\title{
Cognitive stimulation therapy: training, maintenance and implementation in clinical trials
}

\author{
This article was published in the following Dove Press journal: \\ Pragmatic and Observational Research \\ 5 April 2014 \\ Number of times this article has been viewed
}

\section{Zunera Khan \\ Anne Corbett \\ Clive Ballard \\ Wolfson Centre for Age-Related Diseases, King's College London, Guy's Campus, London, UK}

Correspondence: Clive Ballard Wolfson Centre for Age-Related Diseases, King's College London, Guy's Campus, London, SEI IUL, UK Tel +440207848 8054

Fax +440207848 65I5

Email clive.ballard@kcl.ac.uk
Abstract: There are around 35 million people worldwide with dementia, more than half of whom have Alzheimer's disease (AD). Presently there are only four licensed pharmacological treatments available for treating the neuropsychological symptoms of AD. These include cholinesterase inhibitors, licensed for the treatment of people with mild to moderate AD, and an $\mathrm{N}$-methyl-D-aspartate antagonist (memantine) licensed for the treatment of people with moderate to severe AD. These treatment options have modest symptomatic benefits for at least 6 months and possibly for 2 years or longer. Increasing evidence from randomized controlled trials has shown the potential value of cognitive training and cognitive rehabilitation in people with AD. There is a good evidence base to support the use of cognitive stimulation as a nonpharmacological treatment approach for people with $\mathrm{AD}$, of which the most promising is cognitive stimulation therapy (CST). CST has shown benefits for cognition and well-being in people with dementia across a number of randomized controlled trials. There are important key issues related to the use of CST for people with AD, such as long-term benefits, implementation of individualized CST, adjunctive benefits with pharmacological treatments, and optimizing overall implementation of CST. Some of these key issues are already being addressed by ongoing clinical trials. Nevertheless, the strength of the current evidence from randomized controlled trials gives strong support to clinical implementation of CST in practice. Ongoing clinical trials will help to refine and optimize the use of CST in clinical practice.

Keywords: cognitive stimulation therapy, cognition stimulation therapy, intervention, training, dementia, Alzheimer's disease

\section{Introduction to CST in patients with dementia}

Dementia has a vast impact on our health and social care services. There are around 35 million people worldwide with dementia, more than half of whom have Alzheimer's disease (AD). There are currently four licensed pharmacological treatments for AD. Three cholinesterase inhibitors (donepezil, rivastigmine, and galantamine) are licensed for the treatment of people with mild to moderate AD, and an N-methyl-D-aspartate antagonist (memantine) is licensed for the treatment of moderate to severe AD. ${ }^{1}$ All of these treatments confer modest symptomatic benefits for at least 6 months, and possibly for 2 years or longer. In addition, there has been considerable investment in the identification and evaluation of more effective pharmacological therapies, although so far work has had limited success. In contrast, there has been a paucity of research evaluating the potential of nonpharmacological treatment approaches as alternatives or adjuncts to pharmacological therapy. Promising preliminary randomized controlled trials (RCTs) have been conducted emphasizing the potential value of cognitive training 
and cognitive rehabilitation in people with AD. However, the best developed evidence base pertains to cognitive stimulation, defined by Clare and Woods ${ }^{2}$ as "engagement in a range of group activities and discussion aimed at general enhancement of cognitive and social functioning". A recent Cochrane review ${ }^{3}$ identified 15 RCTs of cognitive stimulation in people with mild to moderate dementia, including a total of 718 participants. Overall, the results were encouraging, with significant benefits on cognition and quality of life. It should however be noted that there were no benefits for mood or other neuropsychiatric symptoms. The review also highlighted that many of the included studies used small samples and were of variable quality. These studies included a variety of approaches to cognitive stimulation. The best evidence base with the most robust clinical trials pertains to a specific intervention referred to as cognitive stimulation therapy (CST). CST is delivered according to a specific manual and has been evaluated in clinical trials for people with mild to moderate dementia. This paper focuses on the evidence supporting the use of this specific CST intervention in the treatment of cognitive and functional impairments and the potential impact on quality of life in people with dementia, with some discussion of reality orientation, which was the initial intervention from which CST was developed. Additionally, this paper reviews therapy, training, and challenges in maintenance and implementation of CST in clinical practice.

\section{CST as an intervention}

In many ways, CST is an adaptation of reality orientation. Reality orientation was first developed in the late 1950s, with a focus on trying to address confusion and disorientation. This approach was supported by a number of RCTs of reality orientation indicating a positive impact on orientation, cognition, and independent functioning. ${ }^{4}$ A meta-analysis of six RCTs ${ }^{5}$ indicated significant cognitive and behavioral benefits following reality orientation intervention compared with no treatment or an alternative treatment, confirming the potential for clinical benefit. However, this approach later raised concerns in relation to its clinical significance in dementia care. ${ }^{6}$ For example, when receiving a reality orientation intervention, a person with dementia may be able to correctly state the day of the week but this may not translate into any meaningful impact on their quality of life. These issues outweighed any small significant benefits in dementia care. Although there is some positive evidence for the effect of training and sign-posting upon orientation in a care setting, ${ }^{7,8}$ the effectiveness of reality orientation delivered over a 24 -hour period is challenging to evaluate.
In recent years, CST has evolved from the principles of reality orientation and other interventions promoting cognitive stimulation. CST involves the use of standard tasks which focus on cognitive functions, designed to meet various difficulty levels to suit the individual's ability and rehabilitation. During intervention, a therapist works with the person and their family/carer to identify and set individual goals and implement a plan to achieve these with the aim of improving performance in everyday life. The approach is individualized, building on an individual's strengths whilst compensating for their impairment. The CST protocol developed and optimized by Spector et $\mathrm{al}^{9}$ involves a very specific operationalized approach with 14 sessions. During a typical CST intervention, participants attend a regular session with a group or family caregiver for a minimum of 4 weeks. The session should meet the definition set by Clare and Woods ${ }^{2}$ targeting cognitive and social functioning. Recent study protocols deliver CST training via training days, CST manuals, and DVDs around group activities that aim to provide stimulation for people with mild to moderate dementia. An overview of these sessions is shown in Figure 1.

A number of RCTs have evaluated the benefits of CST in people with mild to moderate dementia (AD, vascular dementia, mixed and other types of dementia) in a range of care settings (community, outpatient clinics, daycare centers, and residential care homes). Outcome measures in these studies have focused on at least one cognitive variable, with additional assessment of other variables, including quality of life, wellbeing, activities of daily living, communication, neuropsychiatric symptoms, and social interaction.

\section{Improved cognition and quality of life Randomized controlled trials}

CST has shown benefits regarding cognition and well-being in people with dementia across a range of RCTs. Aguirre et $\mathrm{al}^{10}$ reported that a CST intervention improved cognition and quality of life independent of whether or not people with dementia were being treated with an acetylcholinesterase inhibitor. A single-blind RCT involving 201 people reported a significant improvement in Mini-Mental State Examination score $(P=0.01)$ and performance on the Alzheimer's disease Assessment Scale Cognition $(P=0.01)$ in a group receiving CST in comparison with a control intervention, and reported additional improvement in quality of life. ${ }^{11} \mathrm{~A}$ further pilot study of CST evaluated a 7-week program of CST delivered twice a week in 45-minute group sessions supplemented by an additional 16 weeks of weekly maintenance CST. The study 
Each cognitive stimulation therapy session follows a general theme, with choices of activities in order to cater to the interests of the group. Members give the group a name. Consistency is created between sessions through using the same warm-up activity, a reality orientation board (containing information about the group), and having a "theme song". Some equipment needs to be purchased or made for the program. Sessions are as follows:

- Physical games

- Sound

- Childhood

- Food

- Current affairs

- Faces/scenes

- Word association

- Being creative

- Categorizing objects

- Orientation

- Using money

- Number games

- Word games

- Team quiz

Additional sessions, outlined in the second cognitive stimulation therapy manual are:

- Useful tips

- Thinking cards

- Art discussion

- Visual clips

- Household treasures

Figure I Structure and content of cognitive stimulation therapy sessions.

reported that improvements in cognition were maintained for around 6 months in people receiving maintenance CST. ${ }^{12}$

There is also some evidence indicating the costeffectiveness of CST based on an RCT comparing 91 people with mild to moderate dementia who received CST and 70 people receiving care as usual. Costs were calculated for the 8 weeks before and the 8 weeks after treatment. There were no cost differences between the groups, but there were quality of life advantages for those receiving CST, indicating that it was a cost-effective intervention. ${ }^{13}$

\section{Qualitative studies}

Qualitative studies have provided further supporting evidence in favor of the use of CST for people with dementia. Spector et $\mathrm{al}^{14}$ investigated the implementation of a group CST intervention in the day-to-day life routine of people with dementia by studying the views of the participants, their carers, and group facilitators. CST was considered to have a positive impact on participants' quality of life in a number of ways. Participants reported feeling more positive, relaxed, and confident following group CST sessions, and felt able to share common difficulties, thus finding it easier to engage in conversation both within and outside of the group sessions. Both participants and their carers felt that participants were engaging more in activities and interacting better with others. Group facilitators also reported some improvements in concentration by individuals attending the group sessions. A third of the carers reported improvements in the person's alertness, concentration, mood, and verbal skills. This study supports the finding reported by Spector et $\mathrm{al}^{11}$ that CST promotes language function, which may lead to overall generalized benefits on quality of life. These outcomes are also in line with previous findings from earlier studies. One study reported that the overall experience of 
CST was seen as emotionally positive, with the majority of participants reporting some change in cognition, ${ }^{15}$ while further work demonstrated improvement in a self-reported measure of quality of life and three key domains of cognitive function, memory, social functioning (relationships with partner/significant other), and activity level (energy and ability to do chores). ${ }^{16}$ This work also showed that improvement in quality of life following CST was significantly correlated with a decrease in symptoms of depression and with improved communication.

There is therefore a strong evidence base in favor of CST as a person-centered approach which aims to preserve cognitive function, enabling optimal engagement in meaningful functional domains and thus improving quality of life. RCTs to date have focused on delivery of CST over relatively short time periods, and have mainly involved people living in residential care settings. There is now a need for further research on the use of CST in the later stages of dementia and across different settings.

\section{Implementation of CST in clinical practice}

One of the largest barriers to the implementation of any psychological or social therapy is the transition from clinical trial to real-life practice. Many nonpharmacological therapies that are successful in RCTs are never widely adopted in clinical practice, and even when that hurdle is overcome, there are significant barriers to effective implementation. For example, for many therapies, implementation manuals are either not available in the public domain or do not contain enough detail to enable the intervention to be delivered optimally, or there are no training or support programs available to ensure fidelity to the intervention. To compound these difficulties, studies have paid little attention to implementation in order to optimize the effectiveness and viability of interventions in real-world clinical or care settings. Importantly, many of these questions have been, or are now being, addressed for CST.

A detailed manual, supported by training courses, is available for CST at moderate cost for a range of health and care professionals, including care workers, occupational therapists, psychologists, and nurses. Training is usually led by a clinical psychologist with extensive experience in CST, who delivers an interactive training forum involving group discussion, exercises, video observation, and role-play, as well as teaching. Training is available in the UK and Europe for up to 25 people per session within a particular setting. With the availability of a detailed manual and a formalized training program, there has been a substantial increase in the use of CST in clinical settings, with around a third of community health teams using this approach in the UK and an increasing number of groups utilizing this approach internationally.

Health care professionals delivering CST have reported the need for management support, online forums, supervision, and additional training with a regular specialist. Many of these features have now been implemented, with online slide sets and programs in place for ongoing support to improve and maintain intervention fidelity in the UK. The effectiveness of this process is currently being evaluated in an ongoing clinical trial.

\section{Critical interpretation}

Overall, the evidence from existing large RCTs using standardized outcome measures suggests that CST is a cost-effective nonpharmacological approach for people with $\mathrm{AD}$ and has significant benefits in terms of cognition and quality of life. CST sessions appear to improve individuals' communication and conversation, which is likely to translate into a generalized benefit on well-being and may explain any related improvement in quality of life. In these studies, the attrition rate has been remarkably low, particularly given the age group of the cohort and nature of the condition.

However, CST can be time-consuming and labor-intensive, and this is an important consideration given that adequate staffing would be vital for its successful implementation in the care home setting. Therefore, use of CST in care home settings may require further evaluation of cost-effectiveness. One further limitation in trials of CST to date has been a lack of long-term follow-up. There is now an ongoing trial looking into long-term implementation of CST. ${ }^{17}$

\section{Ongoing clinical trials and further research Individualized CST}

Currently, there is one ongoing multicenter RCT by a group at University College London, which is looking into the costeffectiveness and benefit of individual home-based CST on cognition and quality of life in people with dementia with a Mini-Mental State Examination score of 10 or above, over 6 months relative to treatment as usual. ${ }^{18}$

\section{Long-term CST}

Another study is evaluating the long-term implementation and use of CST in a Phase IV trial, which will provide an 
important understanding of the barriers and key points related to implementation of CST in practice. ${ }^{17}$

\section{Conclusion: a place for CST in therapy}

In the UK, there is already a national recommendation that CST be made available for people with mild to moderate dementia. ${ }^{19}$ Since 2006, there has been further consolidation of an already strong clinical trial evidence base indicating the clinical benefit and cost-effectiveness of CST. There are important ongoing questions related to key issues such as optimizing long-term benefits, use of individualized CST, adjunctive benefits with pharmacological treatments, and optimizing implementation, many of which are currently being addressed in ongoing clinical trials. However, the strength of the current evidence base is sufficient to endorse clinical implementation, and subsequent work should help to refine and optimize the use of CST in practice. A current urgent priority is to increase the training and support capacity, in particular to enable wider international implementation of CST.

\section{Acknowledgment}

The authors wish to thank the National Institute for Health Research Biomedical Research Centre for Mental Health at South London and the Maudsley NHS Foundation Trust and Institute of Psychiatry, King's College London, for supporting this research.

\section{Disclosure}

The authors report no conflicts of interest in this work.

\section{References}

1. Ballard C, Gauthier S, Corbett A, Brayne C, Aarsland D, Jones E. Alzheimer's disease. Lancet. 2011;377:1019-1031.

2. Woods B, Aguirre E, Spector AE, Orrell M. Can cognitive stimulation benefit people with dementia? Cochrane Database Syst Rev. 2012;2:CD005562.

3. Holden UP, Woods RT. Positive Approaches to Dementia Care. 3rd ed. Edinburgh, Scotland: Churchill Livingstone; 1995.
4. Spector A, Davies S, Woods B, Orrell M. Reality orientation for dementia: a systematic review of the evidence of effectiveness from randomized controlled trials. Gerontologist. 2000;40:206-212.

5. Dietch JT, Hewett LJ, Jones S. Adverse effects of reality orientation. J Am Geriatr Soc. 1989;37:974-976.

6. Hanley IG, McGuire RJ, Boyd WD. Reality orientation and dementia: a controlled trial of two approaches. Br J Psychiatry. 1981;138: $10-14$.

7. McGilton KS, Rivera TM, Dawson P. Can we help persons with dementia find their way in a new environment? Aging Ment Health. 2003;7:363-371.

8. Williams R, Reeve W, Ivison D, Kavanagh D. Use of environmental manipulation and modified informal reality orientation with institutionalized, confused elderly subjects: a replication. Age Ageing. 1987;16:315-318.

9. Spector A, Thorgrimsen L, Woods B, Orrell M. Making a difference: an evidence-based group programme to offer cognitive stimulation therapy (CST) to people with dementia. London, UK: Hawker Publications; 2006.

10. Aguirre E, Hoare Z, Streater A, et al. Cognitive stimulation therapy (CST) for people with dementia - who benefits most? Int J Geriatr Psychiatry. 2013;28:284-290.

11. Spector A, Thorgrimsen L, Woods B, et al. Efficacy of an evidence-based cognitive stimulation therapy programme for people with dementia: randomised controlled trial. Br J Psychiatry. 2003;183:248-254.

12. Orrell M, Spector A, Thorgrimsen L, Woods B. A pilot study examining the effectiveness of maintenance cognitive stimulation therapy (MCST) for people with dementia. Int J Geriatr Psychiatry. 2005;20:446-451.

13. Knapp M, Thorgrimsen L, Patel A, et al. Cognitive stimulation therapy for people with dementia: cost-effectiveness analysis. Br J Psychiatry. 2006;188:574-580.

14. Spector A, Gardner C, Orrell M. The impact of cognitive stimulation therapy groups on people with dementia: views from participants, their carers and group facilitators. Aging Ment Health. 2011;15:945-949.

15. Spector A, Orrell M, Woods B. Cognitive stimulation therapy (CST): effects on different areas of cognitive function for people with dementia. Int J Geriatr Psychiatry. 2010;25:1253-1258.

16. Spector A, Orrell M. Quality of life (QoL) in dementia: a comparison of the perceptions of people with dementia and care staff in residential homes. Alzheimer Dis Assoc Disord. 2006;20(3):160-165.

17. Streater A, Spector A, Aguirre E, et al. Maintenance cognitive stimulation therapy (CST) in practice: study protocol for a randomized controlled trial. Trials. 2012;13:91.

18. Orrell M, Yates LA, Burns A, et al. Individual cognitive stimulation therapy for dementia (iCST): study protocol for a randomized controlled trial. Trials. 2012;13:172.

19. National Institute for Health and Care Excellence and the Social Care Institute for Excellence. Dementia: Supporting people with dementia and their carers in health and social care (CG42). Available from http://www.nice.org.uk/CG042. Accessed October 4, 2013.
Pragmatic and Observational Research

\section{Publish your work in this journal}

Pragmatic and Observational Research is an international, peer-reviewed, open access journal that publishes data from studies designed to reflect more closely medical interventions in real-world clinical practice compared with classical randomized controlled trials (RCTs). The manuscript management system is completely online and includes a very quick and fair peer-review

\section{Dovepress}

system. Visit http://www.dovepress.com/testimonials.php to read real quotes from published authors. 